\title{
An Ultrafast Wide-Band Millimeter-Wave (MMW) Polarimetric Radar for Remote Sensing Applications
}

\author{
A. Y. Nashashibi, Senior Member, IEEE, K. Sarabandi, Fellow, IEEE, P. Frantzis, R. D. De Roo, Member, IEEE, \\ and F. T. Ulaby, Fellow, IEEE
}

\begin{abstract}
With the advent of high-frequency radio frequency (RF) circuits and components technology, millimeter-wave (MMW) radars are being proposed for a large number of military and civilian applications. Accurate and high-resolution characterization of the polarimetric radar backscatter responses of both clutter and man-made targets at MMW frequencies is essential for the development of radar systems and optimal detection and tracking algorithms. Toward this end, a new design is developed for ultrafast, wide-band, polarimetric, instrumentation radars that operate at 35 and $95 \mathrm{GHz}$. With this new design, the complete scattering matrix of a target (magnitude and phase) can be measured over a bandwidth of $500 \mathrm{MHz}$ in less than $2 \mu \mathrm{s}$. In this paper, the design concepts and procedures for the construction and calibration of these radars are described. In addition, the signal processing algorithm and data-acquisition procedure used with the new radars are presented. To demonstrate the accuracy and applicability of the new radars, backscatter measurements of certain points and distributed targets are compared with their analytical radar cross section (RCS) and previously measured $\sigma^{\circ}$ values, respectively, and good agreements are shown. These systems, which can be mounted on a precision gimbal assembly that facilitates their application as high-resolution imaging radar systems, are used to determine the MMW two-way propagation loss of a corn field for different plant moisture conditions.
\end{abstract}

Index Terms-Attenuation, backscattering, clutter, imaging radar, millimeter-wave radar, polarimetry.

\section{INTRODUCTION}

$\mathbf{P}$ ROPER characterization of the backscatter responses of both clutter and man-made targets is best achieved through the use of polarimetric instrumentation radars capable of characterizing the backscattered field by measuring its polarization, magnitude, and phase accurately. Three critical attributes are desired in a millimeter-wave (MMW) instrumentation radar to ensure its versatility: full polarimetry, high-resolution imaging capability, and fast data acquisition. A fully polarimetric radar allows for the measurement of the complex elements of the scattering matrix of a target in a given orthogonal polarization basis (such as the vertical $(\mathrm{V})$ and horizontal $(\mathrm{H})$ polarization basis), from which the radar response in any other transmit/receive polarization configuration can be synthesized [1], [2]. In phenomenological studies of radar backscatter, high-resolution

Manuscript received February 23, 2001; revised June 12, 2002. This work was prepared through collaborative participation in the Advanced Sensors Consortium sponsored by the U.S. Army Research Laboratory under the Federated Laboratory Program, Cooperative Agreement DAAL01-96-2-0001.

The authors are with the Radiation Laboratory, Department of Electrical Engineering and Computer Science, University of Michigan, Ann Arbor, MI 48109-2122 USA.

Publisher Item Identifier 10.1109/TGRS.2002.802462. radar imaging (both in range and along track) can provide insight to the sources responsible for the different scattering mechanisms contributing to the overall backscatter from a given clutter type or man-made target. The high-resolution capabilities can also be used to study the properties of signals propagating through turbulent media such as foliage and rain. It can also form the basis for evaluating the performance of optimal detection and tracking algorithms. While along-track spatial resolution can be achieved through the use of an electrically large aperture, range resolution can only be achieved through the use of a narrow pulse, or equivalently, a wide bandwidth. At MMW frequencies, at least $500 \mathrm{MHz}$ of signal bandwidth, which corresponds to $1-\mathrm{ft}$ range resolution, is required for certain applications and phenomenological studies. It should be noted that the polarimetric measurements need to be performed over the entire signal bandwidth if high-resolution polarimetric images are to be generated. Since the phase of the backscattered signal must be preserved, it is required that the measurement of the scattering matrix elements be completed, over the entire bandwidth, within a period of time sufficiently short to ensure that signal correlation between the scattering matrix elements is maintained. Temporal decorrelation is often due to the relative movement of target or clutter (such as foliage) elements with respect to the radar platform. For example, at $95 \mathrm{GHz}$ (wavelength $\lambda=3.2 \mathrm{~mm}$ ), a $25^{\circ}$ phase error is introduced over $20 \mu$ s for an object moving at a low speed of $20 \mathrm{~km} / \mathrm{h}$.

Vector network analyzer-based instrumentation radars [2], [3] operating in a stepped-frequency continuous-wave mode have been used in the past for characterizing the clutter response at both microwave and MMW frequencies. Although these radars can measure the magnitude and phase of the radar signal over a wide frequency range and with high precision and accuracy, the long data-acquisition time required for these systems ( $0.5 \mathrm{~s}$ per transmitted polarization) renders them less attractive for outdoor polarimetric measurements at MMW frequencies, especially when foliage is the target to measure. Although frequency-modulated continuous wave (FMCW) radars have been proposed for certain applications at MMW frequencies, the difficulty in extracting the phase of the backscattered signal and the linearity constraints on the frequency chirp over the entire band make them less attractive for use as instrumentation radars. Pulse-based polarimetric instrumentation radars have been also used at MMW frequencies [4]. They transmit high-peak power pulses at a single carrier frequency (with the receiver switched off during transmission), and they use I-Q channels for the detection of the magnitude and phase of the 
received signal. A major limitation of pulsed MMW systems is that they have coarse-range resolution because generating pulses on the order of nanoseconds in length is both difficult and expensive. To improve the range-resolution capability of a pulse radar, the carrier frequency can be stepped over a certain bandwidth [5], and then through proper signal processing of the received series of pulses, significant improvement in range resolution can be achieved. However, the time needed to acquire data for generating the scattering matrix with the desired resolution is rather long and is limited by the pulse repetition rate of the system.

The FM-chirped pulse radar, which combines the advantages of FMCW radars (wide-band and low-peak power) and pulse radars (fast data-acquisition and phase-angle measurements), has also been used in many applications [2]. However, linearity constraints on the FM chirp over the desired signal bandwidth and the limited bandwidth of the surface acoustic wave (SAW) devices often used in these system have prohibited their use at MMW frequencies. With recent advances in computer technology and high-speed samplers and analog/digital (A/D) converters, FM-chirped pulse-based instrumentation radars are now feasible at MMW frequencies. The SAW and I-Q channels constituting the conventional receiver in FM-chirped pulse radars can be replaced with a digitizing oscilloscope (DO). Modern DOs use special techniques to combine a set of fast samplers together in order to achieve sampling rates as high as 2 gigasamples/s, so as to sample, in real time, signals up to $1 \mathrm{GHz}$ in frequency. In addition, the development of direct digital synthesizers and chirp generators employing fast D/A converters has permitted the generation of highly linear FMCW waveforms. Through proper frequency multiplication and up-conversion schemes, a rather wide-band highly linear FM-chirped pulse can be generated at MMW frequencies, as will be described later in this paper.

This paper describes a new design of an instrumentation radar, based on the FM-chirped-pulse approach, that overcomes the inherent speed limitations of stepped frequency radars and permits wide-band polarimetric backscatter measurements to be conducted in less than $2 \mu \mathrm{s}$. In this novel and relatively inexpensive design, an ultrafast synthesizer ( $2 \mathrm{~ns} / \mathrm{step}$ ) is used as the linear chirp generator, and a real-time ultrafast multichannel DO (2 gigasamples/s) is used as the intermediate frequency (IF) detector. Using appropriate multiplier, filter, and amplifier combinations, a chirp bandwidth of $500 \mathrm{MHz}$ is generated (with a controllable pulse width of $0.5-16 \mu \mathrm{s}$ ) which is then up-converted to the desired radio frequency (RF) before transmission. The system has the ability to collect internally a sample of each transmitted pulse. This "reference" signal permits continuous monitoring of the system transfer function and is used in subsequent signal processing to enhance signal quality. Using narrow-beam antennas, high-resolution images can be easily generated using this system. The new radar design (high-speed, wide-band, polarimetric) permits phenomenological studies of foliage response to radar signals at MMW frequencies in support of new applications such as the detection of foliage-obscured targets.

In Section II, a detailed description is provided of the new ultrafast wide-band (high-resolution) instrumentation radar

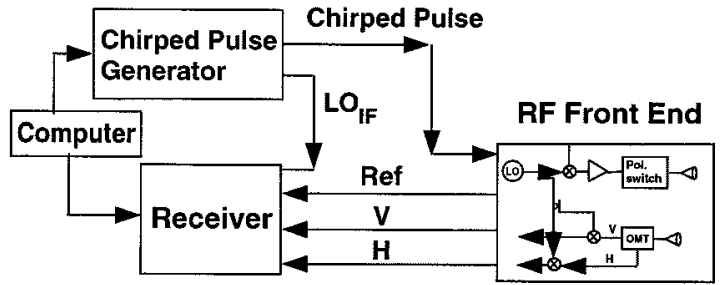

Fig. 1. System block diagram of the new radar design.

structure. The associated signal processing technique and measurement procedure are described in Section III, and in Section IV, system calibration and validation are addressed. Section $\mathrm{V}$ presents an example demonstrating the imaging capabilities of the new system. It also shows a demonstration of the sensitivity of the radar return to the periodic row structure of a corn field, imaged at $35 \mathrm{GHz}$ and $95 \mathrm{GHz}$, as well as deriving the two-way propagation loss inside the corn field.

\section{SYSTEM DESCRIPTION}

At MMW frequencies, it is difficult to generate and detect a wide-band linearly chirped pulse directly. Instead, chirp pulse generation and detection must be conducted at an IF. In this case, the MMW components of the radar are restricted to upand down-conversion of the IF-chirped pulse to MMW-chirped pulses which, in turn, are transmitted/received via an antenna assembly. The ultrafast wide-band system (depicted in Fig. 1) consists of three main modules:

1) linearly chirped pulse generator module;

2) MMW RF frontend module;

3) receiver module.

The system, as it stands, is modular, compact, lightweight, relatively inexpensive, and can be mounted on a gimbal inside a van (for near-grazing imaging applications) or atop a boom truck.

\section{A. Linearly Chirped Pulse Generator Module}

The linearly chirped pulse generator module is designed around a direct digital chirp synthesizer (DCP) unit [6]. The DCP unit consists of the more traditional direct digital synthesizer with a frequency accumulator added in front of the phase accumulator to provide the unit with linear-sweep capability. The computer-controlled DCP unit can be configured so as to generate a pulse, the frequency of which is chirped between two frequencies: a start frequency $f_{\text {start }}$ and a stop frequency $f_{\text {stop }}$, spanning anywhere between $1 \mathrm{MHz}$ and $230 \mathrm{MHz}$. The chirping is accomplished by stepping in frequency at equal increments $\Delta f$ (minimum step size is $29.8 \mathrm{~Hz}$ ) every $2 \mathrm{~ns}$. Both chirp bandwidth and pulsewidth can be controlled via a computer through the proper choice of the programmable $f_{\text {start }}, f_{\text {stop }}$, and $\Delta f$ frequencies. The direct DCP unit requires a $500-\mathrm{MHz}$ reference clock (2-ns period) that is provided through a separate $10-\mathrm{MHz}$ crystal-referenced synthesized source.

A linearly chirped pulse, generated using the DCP unit, can span at most a bandwidth of $230 \mathrm{MHz}$, which might not be sufficient for certain high-resolution MMW radar applications where at least $500 \mathrm{MHz}$ bandwidth is needed. In addition, it is difficult 


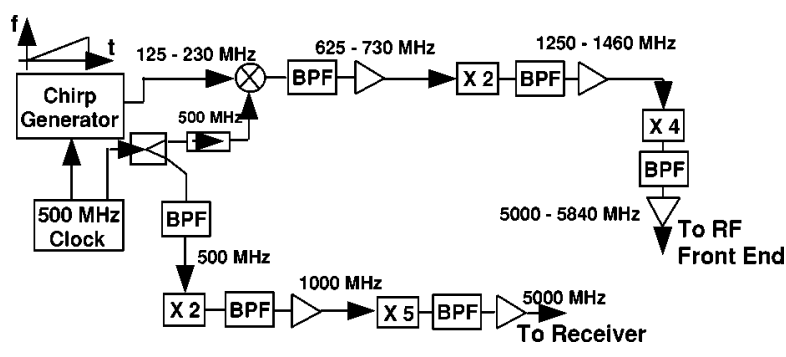

Fig. 2. Block diagram of the linear-chirp pulse generator module.

to up-convert a baseband signal, such as that generated by the DCP unit, directly to MMW frequencies (the lower and upper images after up-conversion to MMW frequencies are, in this case, too close to each other and cannot be isolated efficiently). To overcome these two limitations, a special frequency multiplication circuit (shown in Fig. 2) was designed and constructed for the dual purpose of expanding the chirp bandwidth and translating the chirp from baseband to an IF (C-band in this case) amenable for subsequent up-conversion to MMW frequencies. The circuit also utilized the available $500-\mathrm{MHz}$ clock signal to generate a coherent $\mathrm{CW}$ signal at C-band to be used in the receiver module for down-converting the backscattered chirped pulse to baseband (where detection can be easily accomplished).

In Fig. 2, the 500-MHz clock is the common source for the entire module, thereby ensuring signal coherence. A chirped pulse spanning between 125 and $230 \mathrm{MHz}$ is translated in frequency to $625-730 \mathrm{MHz}$ chirp by mixing it with the $500-\mathrm{MHz}$ reference clock. Then, through a set of properly designed filters, amplifiers, and frequency multipliers, a chirped pulse from 5.0-5.84 GHz is generated. Similarly, a 5.0-GHz reference CW signal is generated from the $500-\mathrm{MHz}$ clock using the $\times 10$ frequency multiplier circuit shown in Fig. 2. Chirp bandwidth less than $840 \mathrm{MHz}$ can be easily achieved by lowering the stop frequency in the DCP unit to less than $230 \mathrm{MHz}$ without altering the circuit in Fig. 2.

The linearly chirped pulse generator module was constructed and tested for spectral purity. Over the entire bandwidth of interest, spurious signals and higher order harmonics were at least $60 \mathrm{~dB}$ below the carrier. Additional details can be found in [8].

\section{B. RF Frontend Module}

The primary function of the RF frontend module is to

1) up-convert an IF-chirped pulse (5.0-5.84 GHz) to MMW frequencies;

2) transmit the MMW-chirped pulse at a given polarization (V or $\mathrm{H})$

3) receive the orthogonal components $(\mathrm{V}$ and $\mathrm{H})$ of the backscatter target response and down-convert them to IF frequencies for subsequent detection.

A block diagram of the University of Michigan's $95-\mathrm{GHz}$ RF frontend module is shown in Fig. 3. In this module, a dual-antenna system (transmit and receive antennas with $2.8^{\circ}$ and $1.4^{\circ}$ beamwidths, respectively) operating in monostatic mode was used. The transmit antenna is fed by an orthomode transducer and a high-isolation (40-dB) high-speed (20-ns) single-pole double-throw (SPDT) switch intended for transmit

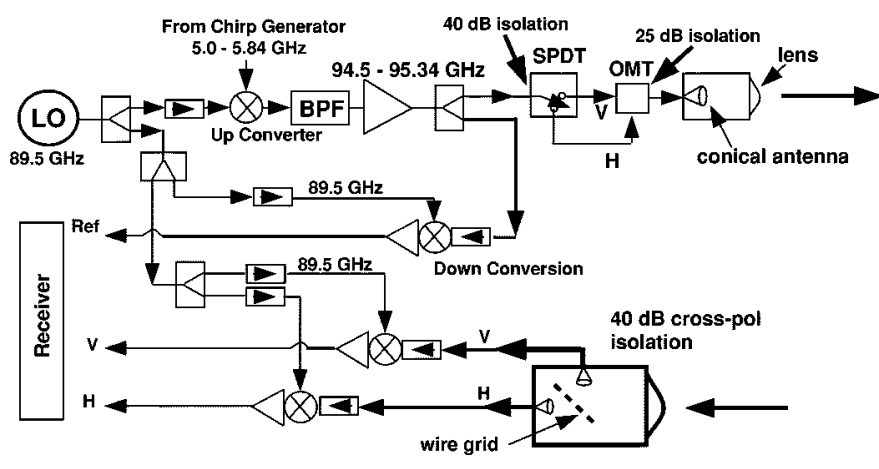

Fig. 3. Block diagram of the 95-GHz RF frontend module.

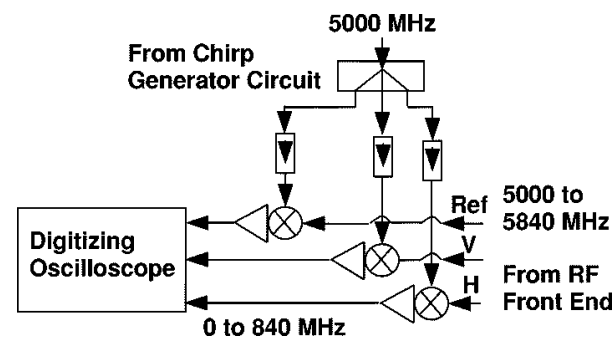

Fig. 4. Block diagram of the receiver module.

polarization selection and emitted about $17 \mathrm{dBm}$ of average power. The receive antenna is a Gaussian optics antenna (high-efficiency and low-sidelobe levels) with an internally installed wire grid polarizer of $40-\mathrm{dB}$ polarization isolation. The wire grid decomposes the received response into its $\mathrm{V}$ and $\mathrm{H}$ orthogonal components. A single $89.5-\mathrm{GHz}$ local oscillator (LO) was used to provide the necessary LO power for both up- and down-conversion, hence maintaining signal coherence in the system. Both $\mathrm{V}$ and $\mathrm{H}$ components of the backscattered response are down-converted simultaneously along with a sample of the transmitted pulse (Ref), as shown in Fig. 3. As mentioned before, the Ref channel plays an important role in signal processing of the backscattered response, as will be demonstrated in Section III. A similar module was constructed at $35 \mathrm{GHz}$ with $\mathrm{LO}$ at $29.8 \mathrm{GHz}$, transmit and receive antennas beamwidths of $4^{\circ}$ and $2^{\circ}$, respectively, and $23 \mathrm{dBm}$ in emitted power.

\section{Receiver Module}

The receiver module (shown in Fig. 4) consists of three C-band mixers followed by baseband amplifiers $(0-1000 \mathrm{MHz})$, which, in turn, feed a four-channel, wide-band DO. The 5.0-5.84-GHz signals of the $\mathrm{V}, \mathrm{H}$, and Ref channels of the RF frontend are down-converted to baseband $(0-840 \mathrm{MHz})$ using the $5.0-\mathrm{GHz}$ reference signal provided by the linearly chirped pulse generator module. The baseband signals are then amplified before being digitized (i.e., sampled and A/D converted) in real time at a rate higher than the Nyquist frequency. The DO used in the new system has 500-MHz bandwidth at baseband, 50-dB dynamic range, and is capable of digitizing signals at a maximum real-time rate of 2 gigasamples/s per channel. Note that the fourth channel of the DO is used for triggering the DO, and the dynamic range of the new radar is limited by the dynamic range of the DO. 
During a given data-acquisition exercise (in which the DO will digitize continuously until the allocated memory storage is full) the radar system will cycle between three basic steps:

Step 1) generate and transmit a linearly chirped pulse;

Step 2) digitize the received radar backscatter response;

Step 3) switch to the other transmitted polarization between consecutive pulses.

It should be noted that the "listen" time between successive pulses, where no signals are transmitted, should be proportional to the round-trip time necessary for the latest part of the chirped pulse to return from the farthest target of interest. Since the DCP unit does not have the capability to switch off on its own for a prescribed time proportional to the desired "listen" time before retransmitting once again, a programmable pulse delay and control circuit was designed and built for this task. For details on the control circuit design, the reader is referred to [8]. The "listen" time can be set so as to detect targets $300 \mathrm{~m}$ away. However, considering the emitted power of the new RF frontends and the weak MMW backscattering coefficients of clutter at near-grazing incidence, the operational range of the system in the field has been between 10 and $50 \mathrm{~m}$.

It should be noted that the system design described above is not restricted to MMW radars. In fact, ultrafast wide-band polarimetric instrumentation radars operating at any frequency band can be constructed easily using the present design. This can be achieved by simply replacing the $95-\mathrm{GHz} \mathrm{RF}$ frontend with other frontends while retaining the chirped pulse generator module, the receiver module, and the control circuit. Both $35-\mathrm{GHz}$ and $95-\mathrm{GHz}$ frontends were used together in recent outdoor measurements. Four IF SPDT computer-controlled switches were used to alternate between the IF ports of the $35-\mathrm{GHz}$ and $95-\mathrm{GHz}$ frontends as the two frontends were imaging a distributed target.

\section{Measurement And Signal Processing Procedures}

The measurement procedure can be summarized as follows. First, the start, stop, and increment step frequencies of the DCP unit are set. Then, the switch-off time of the pulse delay and control circuitry (described in detail in [8]) is set, based on the maximum range of interest; then, the SPDT transmit polarization switch is set to V polarization; and finally the DO is armed. Once the measurement process is initiated by the computer, the chirp generator is switched on, and the DO is triggered to sample signals from the $\mathrm{V}, \mathrm{H}$, and REF channels. At the end of the chirp, the chirp generator is automatically switched off for the prescribed "listen" time, and the polarization switch is switched to the other polarization while the DO continues to sample the received signals. The chirp is then activated again, and the process continues automatically until the DO data storage memory is full. At this time, the computer stops the chirp, resets the polarization switch, transfers the sampled data from the DO data bank, and processes the data. Depending on the pulse length and the switch-off time selected, anywhere from one to several pairs of transmitted pulses can be measured (corresponding to one or several scattering matrices for each target present in the signal propagation path).
The system, as it stands, can collect the scattering matrix of a target, in a single shot, within $2 \mu \mathrm{s}$, which is what is important from a phenomenological studies point of view. The fact that this relatively inexpensive system uses off-the-shelf equipment, namely, a general-purpose $\mathrm{DO}$, forces postacquisition processing of the data to be carried out inside the computer. Currently, the raw data of a scattering matrix is moved to the computer, processed, and stored to the hard disk in less than $2 \mathrm{~s}$, using a computer with a $400-\mathrm{MHz}$ Pentium processor.

\section{A. Signal Processing}

As mentioned before, long pulses with wide-band chirps are employed in the proposed radar design. Whether the targets are close or far away from the radar, the radar return at any instant of time will be a superposition of fields scattered from targets that are located at different ranges and illuminated by different portions of the transmitted pulse (i.e., at different frequencies). Nevertheless, at the end of the DO operation, each target would have been illuminated by the entire transmitted pulse, and its backscatter response would have been detected. In effect, the information about the scattering properties of a given target and its location in range are distributed over the entire received return. Further processing of the sampled data is needed.

Let the output of the reference channel be denoted by $R_{\text {ref }}(t)$. Similarly, let the outputs of the V-and H-receiver channels be denoted by $R_{i j}(t)$ (where $i$ refers to the received $v$ - or $h$-polarized channels, and $j$ refers to the transmitted $v$ or $h$ polarization). The standard approach in processing the radar return (due to a chirped pulse) is to pass it through a filter $h(t)$, the bandwidth of which is equal to the inverse of the pulse duration [7]. Mathematically, the output of the filter $s_{i j}(t)$ is the result of a convolution process between the signal and the filter $s_{i j}(t)=R_{i j}(t) * h(t)$ in the time-domain. In the frequency domain, the output can be expressed as the multiplication of the frequency response of the signal with that of the filter $S_{i j}(f)=$ $\tilde{R}_{i j}(f) \cdot H(f)$. It can be shown that a maximum signal-to-noise ratio (SNR) can be achieved when the filter is proportional to the complex-conjugate of the signal itself $H(f)=K \tilde{R}_{i j}^{*}(f)$, where $K$ is a constant [7]. The filter in this case is called a "matched" filter. It should be noted that, inherent in the assumptions leading to the derivation of the matched filter (with maximum SNR) is that the radar system is ideal.

As mentioned before, the signal in the reference channel is a faithful representation of the transmitted pulse. Hence, $\tilde{R}_{\text {ref }}^{*}(f)$ can be used as the matched filter in computing $S_{i j}(f)$

$$
S_{i j}(f)=\tilde{R}_{i j}(f) \cdot \tilde{R}_{\mathrm{ref}}^{*}(f) .
$$

The measured radar return from a metallic sphere was processed using (1), and its inverse fast Fourier transform (IFFT) is plotted with a dashed line in Fig. 5. It is observed that applying the matched filter directly has resulted in a higher noise floor and higher sidelobe levels than expected. This can be attributed to the fact that the magnitudes of both $\tilde{R}_{v v}(f)$ and $\tilde{R}_{\text {ref }}^{*}(f)$ are not constant; instead, they are functions of frequency. To alleviate this problem, a special window function needs to be designed and implemented. Of course, the inclusion of a window function will inevitably result in a modified matched filter $H(f)=$ 


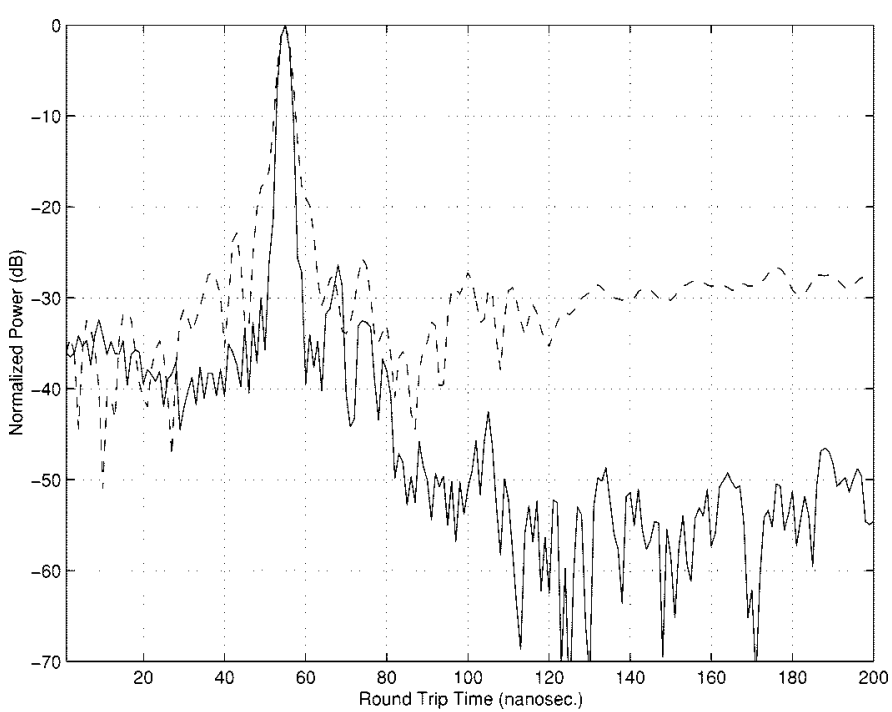

Fig. 5. Measured system responses due to backscatter by a metallic sphere, when processed according to the expressions in (1) and (2). The dashed line refers to (1), while the solid line refers to (2).

$\tilde{R}_{\text {ref }}^{*}(f) \cdot W(f)$ that does not necessarily provide the optimum SNR. A radar system typically contains several time-variant devices, such as amplifiers and mixers; hence, the window function must be updated periodically to account for temporal variations in the transfer function of the system.

In this paper, we propose an alternative approach, namely, that $S_{i j}(f)$ is computed using the reference division, as given by the following expression:

$$
S_{i j}(f)=\frac{\tilde{R}_{i j}(f)}{\tilde{R}_{\mathrm{ref}}(f)}
$$

applied only over $f$ within the desired bandwidth; otherwise $S_{i j}(f)=0$. In other words, the matched filter is replaced with $1 / \tilde{R}_{\text {ref }}(f)$. In this approach, the phase conjugation of the signal, which is necessary for proper focusing of different target returns, is achieved, while the magnitude variations over frequency are corrected at the same time. As a result, an improved time-domain response is obtained (see the solid line in Fig. 5). In addition, any temporal variations in the transmitted pulse will be corrected instantaneously.

A flowchart that demonstrates the signal processing procedure being proposed for processing the data is shown in Fig. 6. In this procedure, the FFT is applied to the sampled data first. Then, the resulting frequency responses of the $\mathrm{V}$ - and $\mathrm{H}$-receiver channels are computed using the expression in (2) at every point within the measured bandwidth. If desired, $S_{i j}(f)$ can be calibrated against the measured frequency response of a calibration target (metallic sphere or trihedral). In addition, a high-resolution time-domain response of a distributed target illuminated by the radar antenna can be obtained by first multiplying $S_{i j}(f)$ with an appropriate window function (to suppress sidelobes), and then performing an IFFT on the product. In cases where the frequency response of a particular target within the radar beam needs to be isolated from that of other targets, software gating on the target's time-domain response should be performed first.
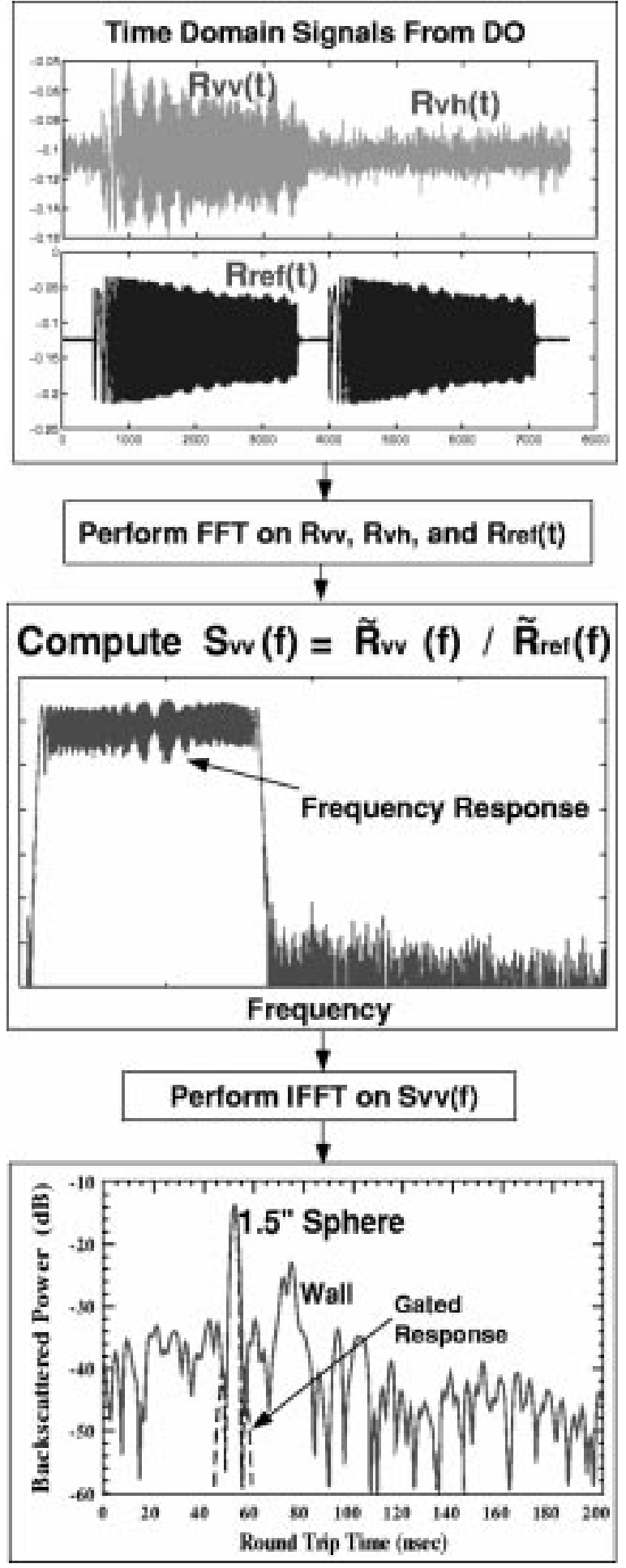

Fig. 6. Flowchart demonstrating the procedure that was used to process the acquired data.

Then, the frequency response of the target can be retrieved from the gated response through an additional FFT step.

The new system is stable enough so that signal coherence between consecutively transmitted pulses is maintained. This is critically important for both the scattering matrix measurement and for cases where coherent subtraction and/or coherent averaging of radar returns is needed. Coherent subtraction is often 


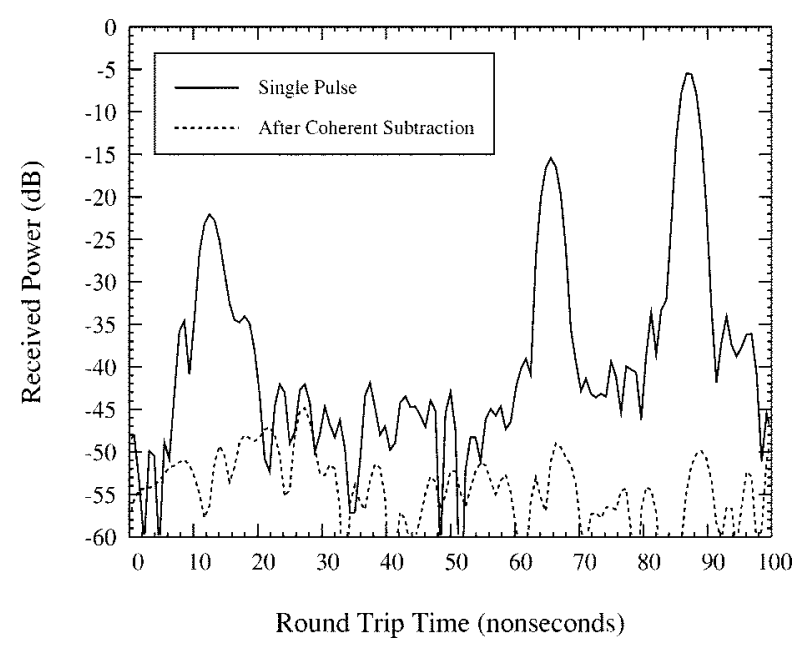

Fig. 7. Comparison between the radar return due to a single pulse (solid line) and the result of coherently subtracting the radar returns of two consecutive pulses (dotted line).

needed in situations where the response of an unwanted background perturbs that of a desired target. In this case, the background is measured first; then, its frequency response is coherently subtracted from the response of the "target + background" case. In Fig. 7, the time-domain response of two point targets due to a single pulse (solid line) is compared with the coherently subtracted responses of the same targets due to two consecutively transmitted pulses (dotted line). It should be noted in this case that the residue from the coherent subtraction is $50 \mathrm{~dB}$ lower than the strongest target return and is limited by the DO dynamic range $(50 \mathrm{~dB})$. Additional tests of the new system have yielded the same level of signal coherence for periods exceeding one hour in an indoor setting (i.e., at a fixed ambient temperature). In cases where weak target returns are observed, coherent averaging of sequentially transmitted pulses can be applied to improve the SNR by reducing the effects of the system's noise. We were able to achieve about $9 \mathrm{~dB}$ improvement in SNR by averaging the return from a small sphere over eight consecutively transmitted pulses (SNR improvement using coherent averaging is proportional to $10 \log _{10} N$ with $N=8$ ).

\section{SyStem CALIBRATION AND VALIDATION}

Characterization of measurement accuracy and precision is critical for any meaningful phenomenological radar measurement. Imperfections in the radar system components, such as antenna polarization contamination (coupling between the orthogonal polarization ports of the antenna) and channel imbalances (variations in magnitude and phase of the system transfer function for different ports of the receiver) can lead to serious errors in the measured scattering matrix. The role of a calibration procedure is, then, to remove the systematic errors from the measured target response. A number of calibration techniques have been reported in the literature, some of which were intended for coherent-on-receive radar systems [4], [9], while others were intended for coherent systems [10]-[14], such as the radar system proposed in this paper. In these calibration techniques, one or more calibration targets with known radar cross sections (RCS) are measured, from which systematic errors are quantified and thus removed. The number of calibration targets needed and the complexity of a chosen calibration procedure depend mostly on the radar architecture and the required measurement accuracy. Since the MMW RF frontends considered in this paper possess high-polarization isolation in their antenna assemblies (25 $\mathrm{dB}$ and $30 \mathrm{~dB}$ cross-polarization isolation for the $35-\mathrm{GHz}$ and $95-\mathrm{GHz}$ RF frontends, respectively), the isolated antenna calibration technique (IACT) introduced in [13] was used to calibrate the systems.

The validity of the IACT technique for calibrating the scattering matrices measured by the new system was examined. A 2 -in diameter sphere and a $20^{\circ}$ tilted dihedral were used as the calibration targets. A conducting cylinder with a diameter of $0.23 \mathrm{~cm}$ and a length of $3.665 \mathrm{~cm}$ was chosen as a test target. The cylinder was oriented at an angle of $43^{\circ}$ from vertical in a plane normal to the direction of incidence. Target orientation was facilitated by an elevation-over-azimuth positioner. The measured scattering matrix of the cylinder was compared with that computed using a method-of-moments (body-of-revolution) code. Considering the number of degrees of freedom in orienting the cylinder and radar platforms, excellent agreement was achieved between the theoretical and measured scattering matrix elements of the cylinder. This comparison is shown in Fig. 8 where maximum discrepancies of about $1.0 \mathrm{~dB}$ in magnitude and $\pm 10^{\circ}$ in phase were observed. Similar results were observed for the $95-\mathrm{GHz}$ scatterometer. These measurements were repeated many times, and consistent results were achieved.

To demonstrate the accuracy of the new ultrafast wide-band radar system in the context of distributed target measurements, a dry smooth asphalt surface with an rms height $s=0.34 \mathrm{~mm}$ and correlation length $l=4.2 \mathrm{~mm}$ was measured using both the new $35-\mathrm{GHz}$ and $95-\mathrm{GHz}$ systems. The same surface was measured two years earlier using a network analyzer-based polarimetric scatterometer operating at $95 \mathrm{GHz}$ [15]. The two experiments were conducted from different platforms and by different individuals using different data acquisition, control, and processing software. In addition, calibration of the network analyzer-based system was performed following the technique developed in [9], whereas the IACT calibration technique was used for the new system. In Fig. 9, the copolarized and cross-polarized backscattering coefficients of the asphalt surface from the two experiments are plotted as a function of the incidence angle $\theta$ (nadir looking is at $\theta=0^{\circ}$ ). Excellent agreement was observed between the two 95-GHz datasets, indicating that the new system's performance (which is representative of the quality of its design, signal processing techniques, and calibration) in characterizing the radar response of clutter, as well as point targets, is comparable with that of currently available scatterometers.

\section{EXAmple: Phenomenology of MMW BACKSCATTER FROM CORN FIELD}

The new radar system was operated in an imaging mode as part of an effort to characterize the polarimetric radar backscatter response of a corn field at near-grazing incidence. Radar images of the corn field were acquired over a period extending from late July (plants were fully grown and green) to late October (plants were fully dry). The corn stalks were 


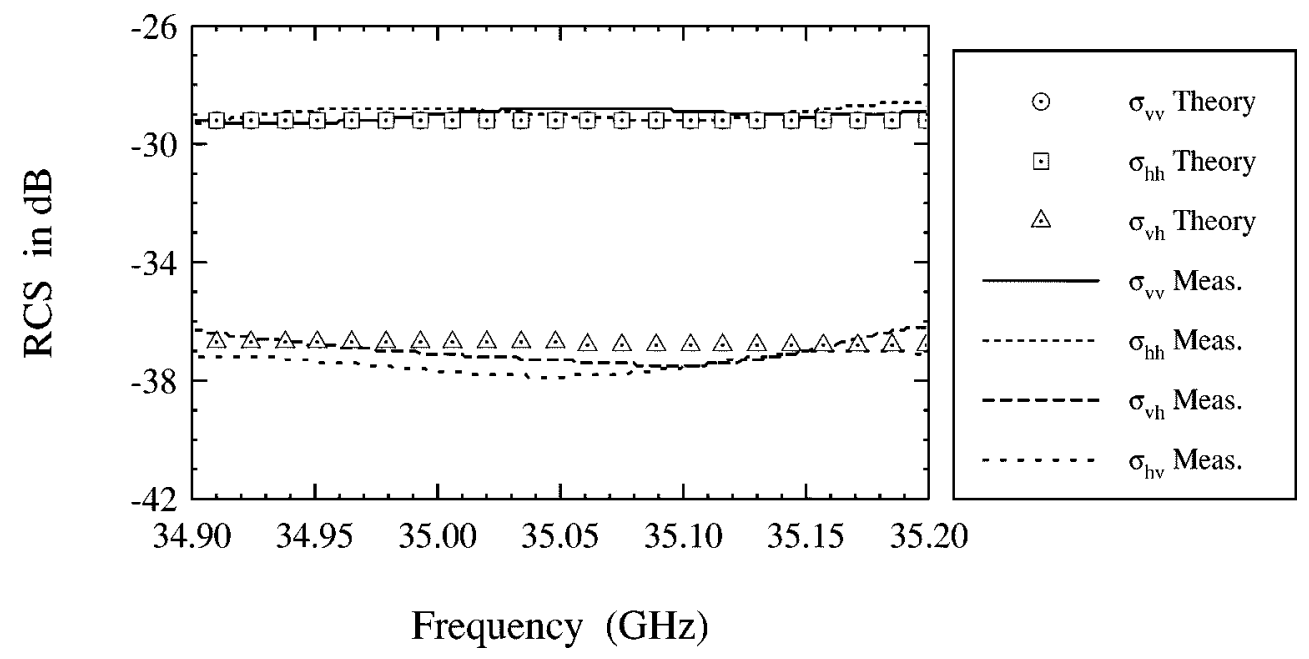

(a)

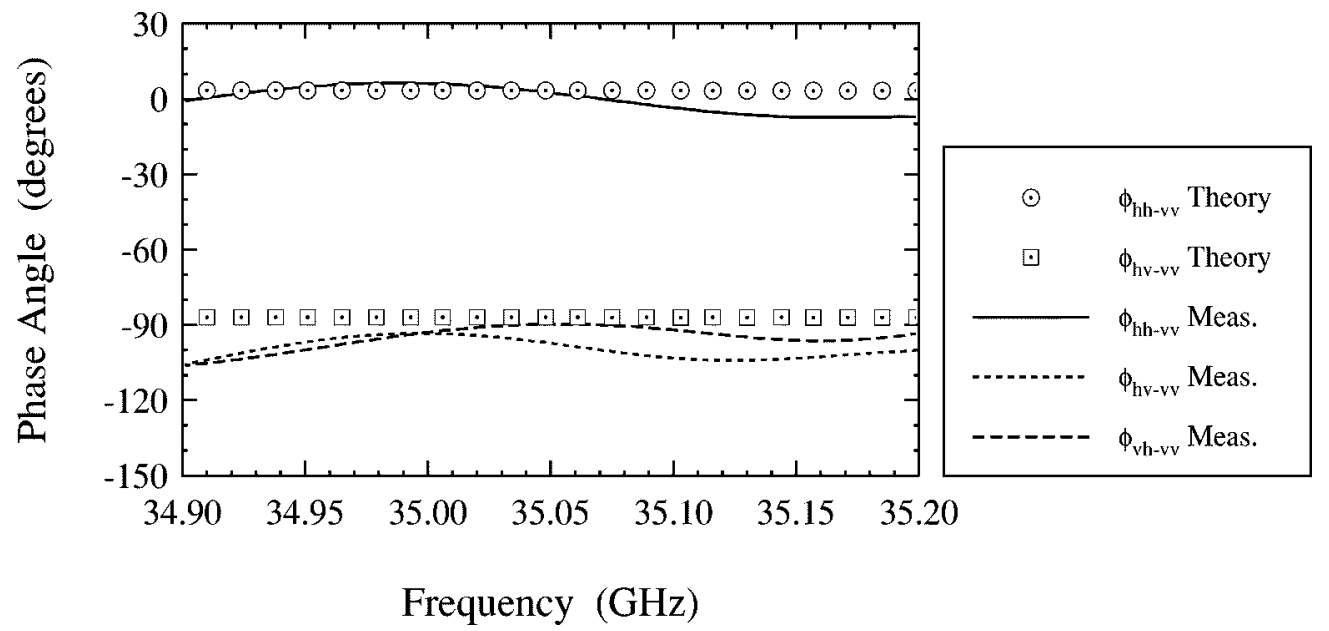

(b)

Fig. 8. (a) Magnitude and (b) phase difference of the scattering matrix elements of a metallic cylinder $(3.665 \mathrm{~cm}$ in length and $0.23 \mathrm{~cm}$ in diameter) oriented at $43^{\circ}$ from vertical.

Dry Smooth Asphalt Surface

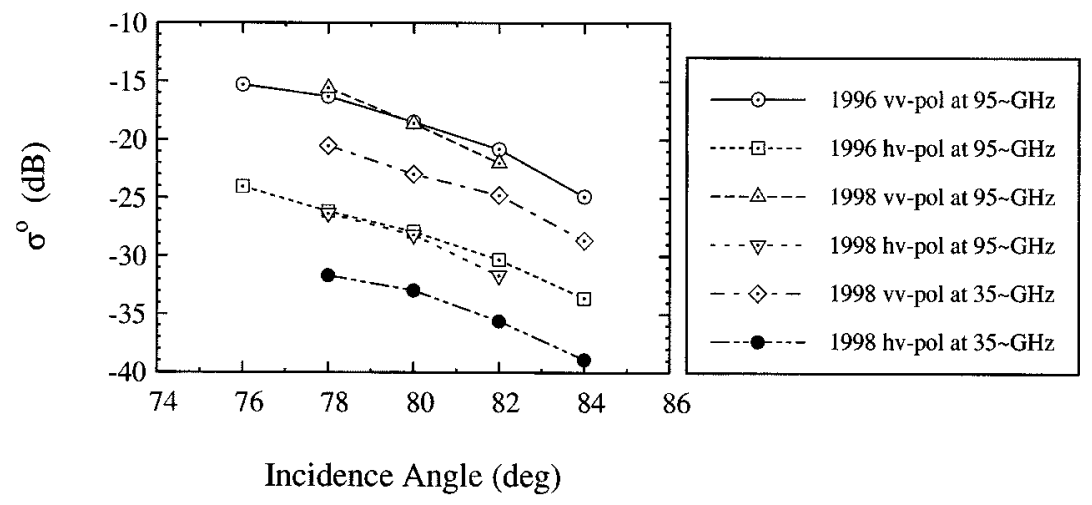

Fig. 9. Backscattering coefficients of a dry smooth asphalt surface measured once using a network analyzer-based scatterometer operating at 95 GHz and measured one more time two years later using the new ultrafast wide-band scatterometers at $35 \mathrm{GHz}$ and $95 \mathrm{GHz}$. The rms height and correlation length of the asphalt surface were $0.34 \mathrm{~mm}$ and $4.2 \mathrm{~mm}$, respectively.

planted in rows with an average distance between rows of $95 \mathrm{~cm}$. The average distance between any two adjacent plants within a row was $20 \mathrm{~cm}$, and the average corn plant height was $2.8 \mathrm{~m}$. Ground truth data of the corn field (such as stalk height, leaf number density, leaf width and length, and gravimetric moisture contents of foliage and soil) were collected at the beginning of each measurement. It was observed that over the measurement period (spanning 80 days), only the gravimetric moisture contents of both the corn plants and the soil underneath it have varied significantly. Hence, it is expected that any 


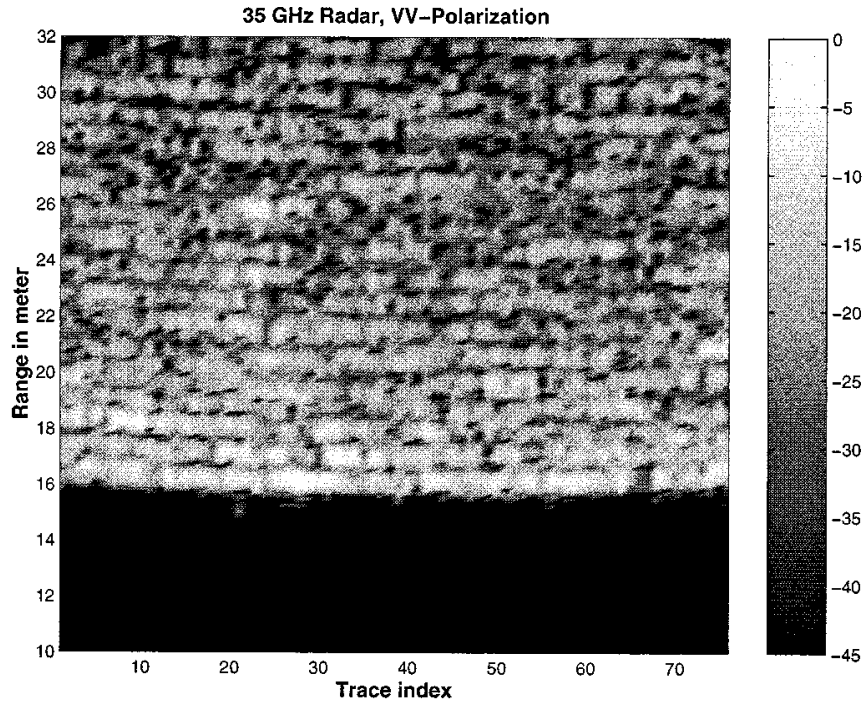

Fig. 10. VV-polarized near-grazing radar image of a corn field measured at $35 \mathrm{GHz}$. The normalized received power is plotted versus range in meters.

temporal variations in the radar response can be related directly to variations in one or both of these quantities. During the radar measurements, the $35-\mathrm{GHz}$ and $95-\mathrm{GHz}$ radar systems, the beamwidths of which are $2^{\circ}$ and $1.4^{\circ}$, respectively, were first oriented at grazing incidence and were positioned at a fixed distance $(16 \mathrm{~m})$ away from the edge of the corn field, where they performed azimuthal scans that were perpendicular to the rows in the corn field. In each scan, between 50 and 80 statistically independent spatial samples were measured. An example of the radar image generated at $35 \mathrm{GHz}$ using the new system is shown in Fig. 10. In this image, the 35-GHz radar signal was able to penetrate through the corn field and detect the periodicity characteristic of the corn field structure. This pattern was also observed at $95 \mathrm{GHz}$, for corn plants under both dry and green conditions. Prior to these observations, the general understanding of the phenomenology of wave propagation through foliage has been that severe attenuation is expected as MMW signals propagate through foliage, and as a result, MMW remote sensing through foliage is impractical. The image in Fig. 10 clearly demonstrates the potential of MMW signal penetration through foliage. It has also led to further research into the propagation characteristics of MMW signals in foliage at both nadir and grazing incidences [16], [17], and into the detectability of targets obscured by foliage [18].

In the case of the corn field under investigation here, we have examined the dependence of both the backscattering coefficients and the attenuation rate on plant moisture content. It was observed that the copolarized backscattering coefficients $\sigma_{v v}^{\circ}$ and $\sigma_{h h}^{\circ}$ were highly correlated in magnitude to within $1 \mathrm{~dB}$ of each other for all acquired data and at both radar frequencies. It is worth noting that, although the corn plant has a distinctive vertically aligned component such as the stalk, its vv-polarized backscatter response is equal to that for hh polarization. This can be explained by noting that, at MMW frequencies, the corn plant parts are many wavelengths in size. In this case, scattering from these parts falls into the geometric optics regime where vv-

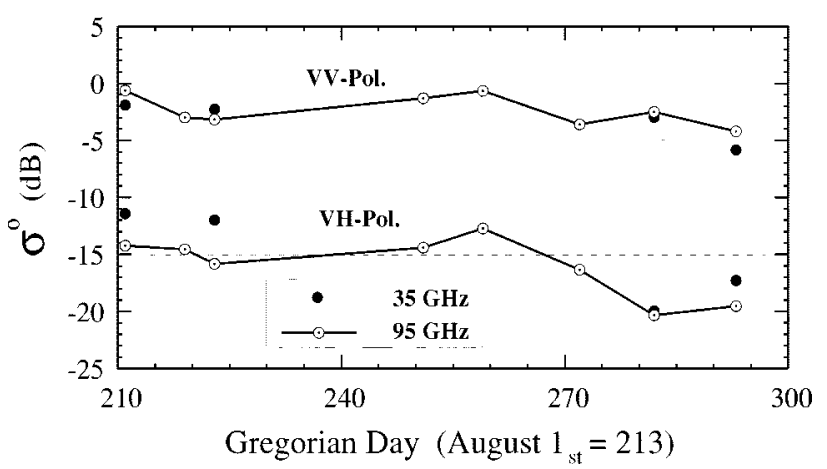

(a)

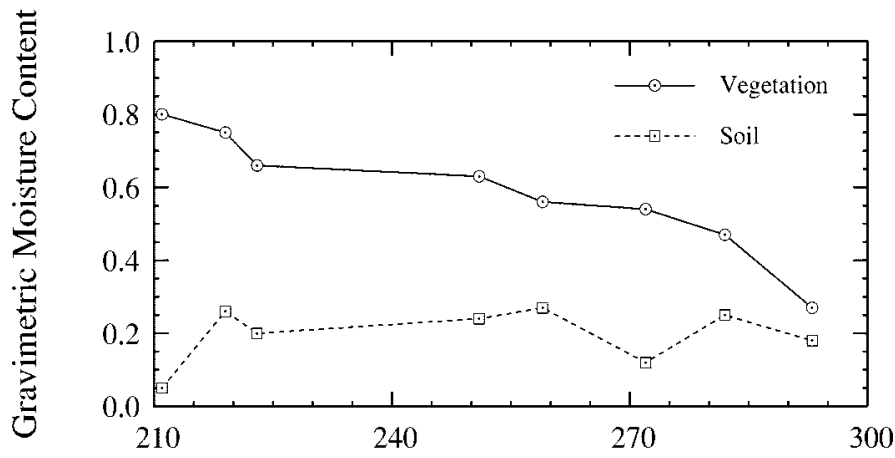

Gregorian Day (August $1^{\text {st }}=213$ )

(b)

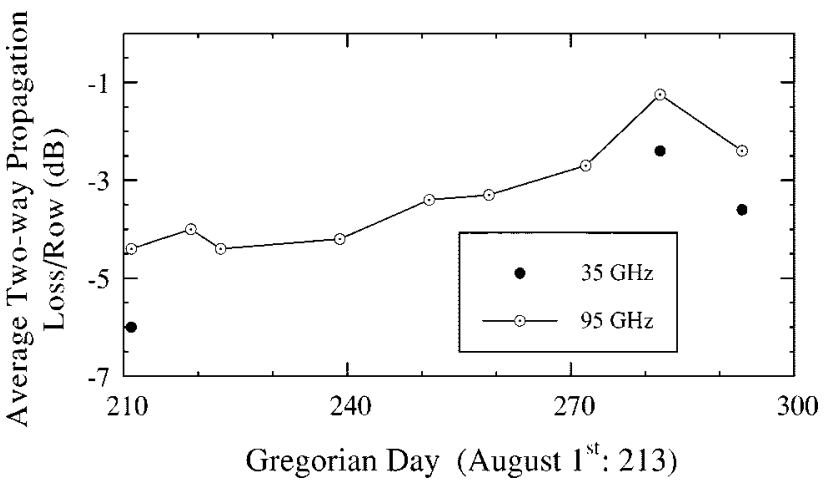

(c)

Fig. 11. Temporal variations of (a) the backscattering coefficients $\sigma_{v v}^{\circ}$ and $\sigma_{v h}^{\circ}$, (b) the gravimetric moisture content of the corn plants and soil, and (c) the average two-way propagation loss per row in the corn field.

and hh-polarized responses of plates (most scattering parts of a corn plant can be approximated as high dielectric plates) are approximately equal. In Fig. 11(a), the backscattering coefficients $\sigma_{v v}^{\circ}$ and $\sigma_{v h}^{\circ}$ are plotted as a function of the Gregorian dates on which the measurements were conducted. For comparison purposes, the gravimetric moisture contents of the corn plants and the soil are plotted in Fig. 11(b). Fig. 11(a) indicates that the copolarized responses at $35 \mathrm{GHz}$ and $95 \mathrm{GHz}$ are essentially the same. In addition, the total dynamic range of both $\sigma_{v v}^{\circ}$ and $\sigma_{v h}^{\circ}$ is around $5 \mathrm{~dB}$ at both frequencies. This narrow dynamic range spanned the entire measurement period, where the moisture content of the corn plants varied between 0.8 and 0.25 . Considering the narrow beamwidths of the system and the geometry of the measurement setup, it can be concluded that the soil moisture did not play a direct role in the measured backscatter response. 


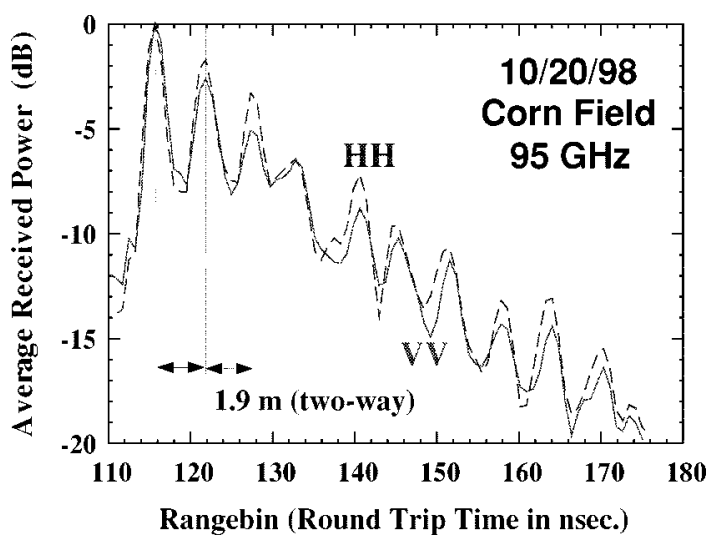

Fig. 12. Average time-domain return from dry corn at $95 \mathrm{GHz}$.

In addition, it is observed that the corn field, when moist, depolarizes about $3 \mathrm{~dB}$ more at $35 \mathrm{GHz}$ as compared to $95 \mathrm{GHz}$. However, as plants dry out, they have essentially the same depolarization strength at both frequencies.

To determine the attenuation rate for this corn field for a given scan, an average radar return was calculated first by averaging the backscatter returns at every rangebin over all measured spatial samples in that scan. An example of the averaged return as a function of the round-trip time is shown in Fig. 12 for the vv- and hh-polarized returns of a dry corn field measured at $95 \mathrm{GHz}$. It should be noted that, since scanning was done perpendicular to the rows in the field, the process of averaging the samples did not destroy the periodicity in the averaged return. Since no physical anomalies were observed in the corn field, it can be safely assumed that the field is a statistically homogeneous random medium. In this case, the reduced return from any given row is the result of the round-trip attenuation suffered by the radar signal as it propagated through the rows that were present between the radar and the row of interest. This attenuation can be attributed to both scattering and absorption in the corn plants. The ratio between the received powers of successive peaks was used to compute the average round-trip propagation loss per row in this corn field. Fig. 11(c) demonstrates the trend in the average round-trip propagation loss per row of the corn field during its life cycle at both 35 and $95 \mathrm{GHz}$. This figure clearly demonstrates the impact of the plant moisture content on the penetrability of the corn field. It is worth noting that the ratio between the maximum and minimum gravimetric moisture contents of foliage measured ( 0.8 and 0.25 , respectively) is about $5 \mathrm{~dB}$, which in turn is equal to the dynamic ranges for both the backscattering coefficients and the two-way propagation loss (see Fig. 11).

\section{CONCLUSION}

A pair of new MMW wide-band polarimetric instrumentation radars were developed at the University of Michigan for remote sensing applications. With the new system design, the scattering matrix of a target can be measured over a bandwidth of $500 \mathrm{MHz}$ in as little as $2 \mu \mathrm{s}$. The short data-acquisition time ensures correct characterization of the complex scattering matrix elements. Details of the system design were discussed along with data acquisition, signal processing, and external calibration techniques. The capability of the new system to properly characterize the backscattering coefficients of distributed targets was demonstrated. In addition, its ability in generating fully polarimetric radar images of $1-\mathrm{ft}$ resolution was demonstrated. Sample images obtained from a corn field show periodicity in the corn row structure and were used to determine the average round-trip propagation loss through the corn field as a function of plant moisture content at both $35 \mathrm{GHz}$ and $95 \mathrm{GHz}$. A narrow dynamic range of about $5 \mathrm{~dB}$ was observed for the backscattering coefficients at both $35 \mathrm{GHz}$ and $95 \mathrm{GHz}$ over the entire measurement period. In addition, the radar return at both radar frequencies was essentially the same when the corn plants were dry.

\section{ACKNOWLEDGMENT}

The authors would like to thank B. Wallace, R. Wellman, E. Adler, and M. Conn of the Army Research Laboratory for their helpful suggestions during the initial phases of the radar design.

\section{REFERENCES}

[1] J. R. Huynen, "Measurement of target scattering matrix," Proc. IEEE, vol. 53, pp. 936-946, 1965.

[2] F. T. Ulaby and C. Elachi, Radar Polarimetry for Geoscience Applications. Norwood, MA: Artech House, 1990.

[3] F. T. Ulaby, M. W. Whitt, and K. Sarabandi, "AVNA-based polarimetric scatterometers," IEEE Antennas Propagat. Mag., vol. 32, pp. 6-17, Oct. 1990.

[4] MAJ. B. Mead, "Polarimetric measurements of foliage and terrain at 225 GHz," Ph.D. dissertation, Univ. Massachusetts, Amherst, 1990.

[5] R. J. Wellman, J. Nemarich, H. Dropkin, D. R. Hutchins, J. L. Silvious, and D. A. Wikner, "Polarimetric monopulse radar scattering measurements of targets at $95 \mathrm{GHz}$," in Proc. AGARD, vol. 501, Sept. 1991, pp. 30-1-30-13.

[6] Sciteq Electronics, DCP-1 Chirp Synthesizer. San Diego, CA: Sciteq Electronics.

[7] N. Levanon, Radar Principles. New York: Wiley, 1988.

[8] A. Y. Nashashibi, K. Sarabandi, P. Frantzis, R. D. De Roo, and F. T. Ulaby, "Design of an ultrafast, wide-band, millimeter-wave, polarimetric, instrumentation radar," Radiation Lab., Dept. Elec. Eng. Comput. Sci., Univ. Michigan, Ann Arbor, MI, Tech. Rep. RL-1006, 2002.

[9] A. Nashashibi, K. Sarabandi, and F. T. Ulaby, "A calibration technique for polarimetric coherent-on-receive radar systems," IEEE Trans. Antennas Propagat., vol. 43, pp. 396-404, Apr. 1995.

[10] R. M. Barnes, "Polarimetric calibration using in-scene reflectors," MIT Lincoln Lab., Lexington, MA, Rep. TT.65, 1986.

[11] M. W. Whitt, F. T. Ulaby, P. Polatin, and V. V. Liepa, "A general polarimetric radar calibration technique," IEEE Trans. Antennas Propagat., vol. 39, pp. 62-67, Jan. 1991

[12] M. W. Whitt and F. T. Ulaby, "A polarimetric radar calibration technique with insensitivity to target orientation," Radio Sci., vol. 25, pp. 1137-1143, Nov./Dec. 1990.

[13] K. Sarabandi, F. T. Ulaby, and M. A. Tassoudji, "Calibration of polarimetric radar systems with good polarization isolation," IEEE Trans. Geosci. Remote Sensing, vol. 28, pp. 70-75, Jan. 1990.

[14] K. Sarabandi and F. T. Ulaby, "A convenient technique for polarimetric calibration of radar systems," IEEE Trans. Geosci. Remote Sensing, vol. 28, pp. 1022-1033, Nov. 1990.

[15] K. Sarabandi, E. S. Li, and A. Y. Nashashibi, "Modeling and measurements of scattering from road surfaces at millimeter-wave frequencies," IEEE Trans. Antennas Propagat., vol. 45, pp. 1679-1688, Nov. 1997.

[16] A. Y. Nashashibi, F. T. Ulaby, P. Frantzis, and R. D. De Roo, "Measurements of the propagation parameters of tree canopies at MMW frequencies," IEEE Trans. Geosci. Remote Sensing, vol. 40, pp. 298-304, Feb. 2002. 
[17] A. Y. Nashashibi, K. Sarabandi, S. Oveisgharan, M. C. Dobson, W. Walker, and E. Burke, "Millimeter-wave measurements of foliage attenuation and ground reflectivity of tree stands at nadir incidence," IEEE Trans. Antennas Propagat. , submitted for publication.

[18] A. Y. Nashashibi and F. T. Ulaby, "Millimeter-wave radar detection of partially obscured targets," in Proc. IEEE AP-S Int. Symp. and USNC/URSI National Radio Science Meeting, Boston, MA, July 9-13, 2001.

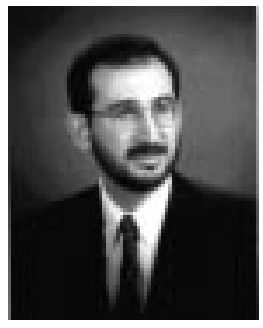

A. Y. Nashashibi (S'82-M'95-SM'01) received the B.S. and M.S. degrees in electrical engineering from Kuwait University, Kuwait, in 1985 and 1988, respectively. He received the Ph.D. degree in electrical engineering from the University of Michigan, Ann Arbor, in 1995.

He is currently an Assistant Research Scientist at the Radiation Laboratory at the University of Michigan. His research interests include microwave remote sensing, polarimetric millimeter-wave radars, calibration and measurement techniques, electromagnetic wave propagation, and scattering in random media.

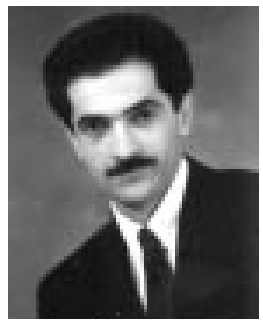

K. Sarabandi (S'87-M'90-SM'92-F'00) received the B.S. degree in electrical engineering from Sharif University of Technology, Tehran, Iran, in 1980 . He received the M.S. degree in electrical engineering (1986), the M.S. degree in mathematics (1989) and the Ph.D. degree in electrical engineering from The University of Michigan, Ann Arbor (1989).

$\mathrm{He}$ is currently the Director of the Radiation Laboratory, as well as a Professor in the Department of Electrical Engineering and Computer Science, at the University of Michigan. His research areas of interest include microwave and millimeter-wave radar remote sensing, electromagnetic wave propagation, and antenna miniaturization. He has 20 years of experience with wave propagation in random media, communication channel modeling, microwave sensors, and radar systems and is leading a large research group that includes two research scientists, ten Ph.D. students, and two M.S. students. Over the past ten years, he has graduated $14 \mathrm{Ph} . \mathrm{D}$. students. He has served as the Principal Investigator on many projects sponsored by the National Aeronautics and Space Administration, Washington, DC; Jet Propulsion Laboratory, Pasadena, CA; The Army Research Office, Research Triangle Park, NC; The Office of Naval Reserach, Arlington, VA; The U.S. Army Research Laboratory, Adelphi, MD; National Science Foundation, Arlington, VA; the Defense Advanced Research Projects Agency, Arlington, VA; and numerous industries. He has published many book chapters and more than 95 papers in refereed journals on electromagnetic scattering, random media modeling, wave propagation, antennas, microwave measurement techniques, radar calibration, inverse scattering problems, and microwave sensors. He has also had more than 200 papers and invited presentations in many national and international conferences and symposia on similar subjects. In the past several years, joint papers presented by his students at a number of symposia (IEEE AP'95,'97,'00, and '01, IEEE IGARSS' 99, IEEE MTTS'01) have received Student Prize Paper Awards. He is also a member of Commission F of URSI and of The Electromagnetic Academy.

Dr. Sarabandi is a Vice President of the IEEE Geoscience and Remote Sensing Society (GRSS), Chairman of the Awards Committee of the IEEE GRSS, and a member of IEEE Technical Activities Board Awards Committee. He serves as Associate Editor of the IEEE TRANSACTIONS ON ANTENNAS AND PROPAGATION and the IEEE SENSORS JOURNAL. He is listed in American Men \& Women of Science Who's Who in America and Who's Who in Electromagnetics. He was the recipient of the highest honor the University of Michigan bestows on a faculty member at the assistant or associate level: the prestigious Henry Russel Award from the Regent of the University of Michigan. In 1999, he received a GAAC Distinguished Lecturer Award from the German Federal Ministry for Education, Science, and Technology, Bonn, Germany, given to about ten individuals worldwide in all areas of engineering, science, medicine, and law. He was also a recipient of a 1996 Teaching Excellence Award from the EECS Department of the University of Michigan.

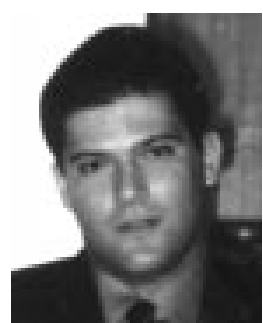

P. Frantzis was born in Nicosia, Cyprus, on December 3, 1971. He received the B.S. degree in electrical engineering from the University of Kansas, Lawrence, in 1997, and the M.S. degree in electrical engineering from the University of Michigan, Ann Arbor, 1999. He is pursuing the MBA degree at Eastern Michigan University, Ypsilanti, MI.

$\mathrm{He}$ is currently working at EMAG Technologies, Inc., Ann Arbor, MI, where he is an R\&D Engineer, Lab Director. His research interests include microwave remote sensing, antenna and radar system design, and calibration of polarimetric SAR systems.

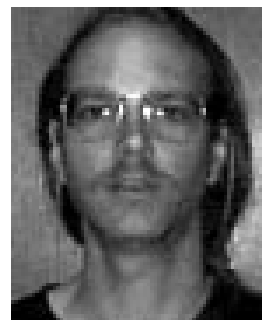

R. D. De Roo (S'88-M'89) received the B.S. degree in Letters and Engineering from Calvin College, Grand Rapids, MI, in 1986, the B.S.E., M.S.E., and $\mathrm{Ph}$.D. degrees from the University of Michigan, Ann Arbor, in 1986, 1989, and 1996, respectively, all in electrical engineering. His dissertation topic was on the modeling and measurement of bistatic scattering of electromagnetic waves from rough surfaces.

$\mathrm{He}$ is currently an Assistant Research Scientist in the Space Physics Research Laboratory, University of Michigan. From 1996 to 2000, he was a Research Fellow at the University of Michigan's Radiation Laboratory, where he investigated the modeling and simulation of millimeterwave backscattering phenomenology of terrain at near grazing incidence. His current research interests include synthetic thinned array radiometry and inversion of soil moisture and vegetation parameters from radar and radiometric signatures of terrain.

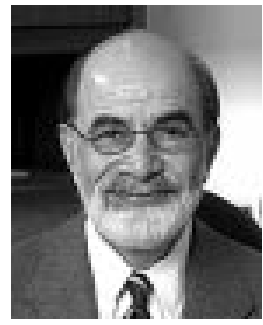

F. T. Ulaby (M'68-SM'74-F'80) received the B.S. degree in physics from the American University of Beirut, Lebanon, in 1964, and the M.S.E.E. and Ph.D. degrees in electrical engineering from the University of Texas, Austin, in 1966 and 1968, respectively.

$\mathrm{He}$ is the Vice President for Research and Williams Distinguished Professor of electrical engineering and computer science at the University of Michigan, Ann Arbor. His current reserarch interests include microwave and millimeter-wave remote sensing, radar systems, and radio wave propagation. He has authored ten books and published more than 500 papers and reports on these subjects.

Dr. Ulaby is the recipient of numerous awards, including the Eta Kappa $\mathrm{Nu}$ Association C. Holmes MacDonald Award as "An Outstanding Electrical Engineering Professor in the United States of America for 1975," the IEEE Geoscience and Remote Sensing Distinguished Achievement Award in 1983, the IEEE Centennial Medal in 1984, The American Society of Photogrammetry's Presidential Citation for Meritorious Service in 1984, the NASA Group Achievement Award in 1990, and the 2000 IEEE Electromagnetics Award. He was President of the IEEE Geoscience and Remote Sensing Society from 1980 to 1982, Executive Editor of IEEE TRANSACTIONS ON GEOSCIENCE AND REMOTE SENSING from 1983 to 1985, and General Chairman of several international symposia. In 1995, he was elected to membership in the National Academy of Engineering, and currently serves as Editor-in-Chief of the IEEE PROCEEDINGS. 